\title{
Cooperative Localization Method for AUV Using Acoustic Communication Measurement
}

\author{
Lichuan Zhang ${ }^{1,2,}$, Mingyong Liu $^{1}$ and Fubin Zhang ${ }^{1}$ \\ ${ }^{1}$ College of Marine, Northwestern Polytechnical University, Xi'an, China \\ ${ }^{2}$ National Key Lab for Underwater Information and Control, Xi'an, China \\ azlc@nwpu.edu.cn
}

\begin{abstract}
Keywords: Autonomous Underwater Vehicle (AUV), Cooperative Navigation, Acoustic communication.
\end{abstract}

\begin{abstract}
In this paper, we propose an algorithm based on double acoustic measurement for cooperative navigation of multiple autonomous underwater vehicles. Research on cooperative navigation of AUV is an important topic to solve the navigation problem in long range and deep sea. We investigate the improvement in navigation accuracy. In the Leader-follow structure, the leader AUV is equipped with high precision navigation system, and the follow AUV is equipped with low precision navigation system. They all are equipped with acoustic device to measure relative location. Traditionally geometry triangulation method is used to calculate the position of follow AUV, the method may cause fault error solution. Double acoustic communication measurement method was designed, which fused the proprioceptive and exteroceptive sensors. The research results prove that the navigation accuracy has been improved effectively.
\end{abstract}

\section{Introduction}

With the development of ocean economic, marine robotics technology has undergone a phase of dramatic increase in capability in recent years. Autonomous Underwater Vehicle (AUV) is an important tool to solve the complex ocean missions. Navigation is one of the key challenges that limit our capability to use AUV to complete ocean mission. There are four traditional methods [1][2] for AUV navigation, 1) Inertial navigation, 2) Acoustic navigation, 3) Earth geophysical navigation, 4) Global navigation system. Those traditional navigation algorithms cannot satisfy the complex ocean operations. Inertial Navigation System (INS) has generally been used for AUV. Although the system can offer good navigation accuracy, it also has several drawbacks. Its error enlarges with time. High level INS is large and expensive, small AUVs cannot afford it. Long Baseline (LBL) is a credible acoustic navigation system, its accuracy can reach several meters, but its operation area limits to a few $\mathrm{km}^{2}$. These traditional navigation systems have no advantage in cooperative mission. We study the cooperative navigation algorithm and localization accuracy of multiple Autonomous Underwater Vehicles. One of the advantages of multiple AUVs system is that AUV can accurately positioning by measuring their relative position and communicating localization information throughout the acoustic network.

Time-synchronized acoustic data and relative range are used to calculate the relative range and update the position of the group vehicles in [3]. In [4] the author develops and testes an algorithm for multiple AUVs with both range measurement and communication. In [5] the author calculates the position with both time synchrony and acoustic communication, and system model is studied. In [6] experimental results of a cooperative navigation method are presented. In [7][8] the author presents EKF method for cooperative navigation system.

This paper deals with a navigation algorithm for multiple vehicles. The leader AUV equips acoustic modem and high accuracy INS. Each fellow AUV equips acoustic modem and dead-reckoning sensor. The system was called Leader-follow cooperative navigation system. Double acoustic measurement algorithm is presented. The advantage of the system is that all fellow AUVs can get high accuracy with low-cost sensors, and can work in any zone of the sea. 


\section{Cooperative Navigation Method}

There are two forms of cooperative navigation. One is parallel form: all of the AUVs have equipped the same sensors. They are equal and can get the information of any other one. The other is Leader-Fellow form: few leader AUVs equip with high level sensors and many follow AUVs equip with low-cost sensors. Follow gets the information from the leader, and then updates its position. This form reduces the cost and reaches the request. So it has been the most important approach to solve the underwater navigation problems.

System Structure. Leader-follow is a new developed cooperative navigation method. The improvement of Leader-follow is that the transponder is fixed on leader AUV. We consider the Leader-Fellow form in the paper. Firstly, the transponder responses the acoustic ping sent by Follow AUV with another frequency ping. When the follow AUV receives the respond ping, it can calculate the relative position between the leader AUV. Then leader AUVs broadcast their true position throughout the acoustic communication link. So the follow AUVs may work out their true position using the relative position and true position.

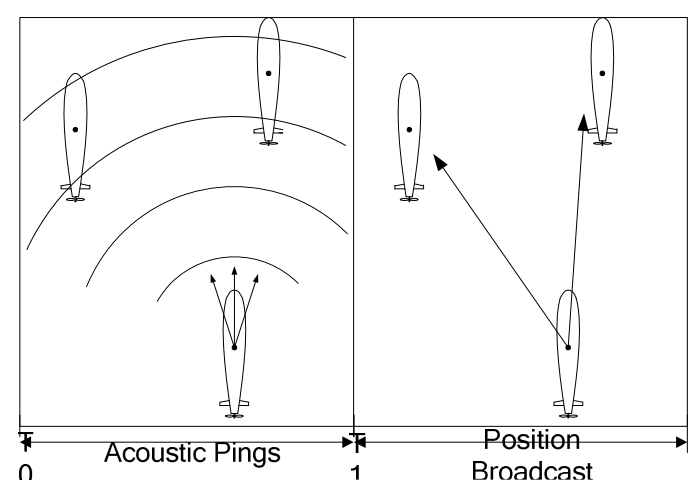

Figure 1. Scheme of Leader-follow structure

System Math Model. Consider the AUV as a point. $\left(x_{k}, y_{k}, z_{k}\right)$ is the trajectory position of AUV at time $k$. Where $x_{k}$ is the longitude in degree, $y_{k}$ is the latitude in degree and $z_{k}$ is the depth in meter. $z_{k}$ can be measured by the depth sensor directly, so in the model we do not need take it into account. The model is described as follows:

$$
\left\{\begin{array}{l}
x_{k+1}=x_{k}+T \cdot V_{k} \cdot \cos \left(\phi_{k}\right) \\
y_{k+1}=y_{k}+T \cdot V_{k} \cdot \sin \left(\phi_{k}\right) \\
\phi_{k+1}=\phi_{k}
\end{array}\right.
$$

Where $T$ is the periods time, $V_{k}$ is the velocity measured with Doppler Velocity Logger (DVL), $\phi_{k}$ is the heading angle which is measured with compass.

In the model, the two measured inputs are modeled as

$$
u_{k}=\left[\begin{array}{c}
V_{k} \\
\phi_{\mathrm{k}}
\end{array}\right]=\left[\begin{array}{c}
V_{m k}-\omega_{v k} \\
\phi_{\mathrm{mk}}-\omega_{\phi k}
\end{array}\right]
$$

Where $\omega_{v k}$ and $\omega_{\phi k}$ are assumed to be independent white Gaussian noise processes with zero mean and known covariances $\sigma_{v_{k}}^{2}$ and $\sigma_{\phi_{k}}^{2}$ respectively.

The model can be described shortly as follow:

$$
X_{k+1}=f\left(X_{k}, u_{k}, w_{k}\right)=X_{k}+\Gamma\left(u_{k}+w_{k}\right)
$$

Where $X_{k}=\left(x_{k} y_{k} \phi\right)^{T}$ represents AUV's position at time $k, \Gamma\left(u_{k}+w_{k}\right)$ is a nonlinear item, $w_{k}$ is white Gaussian noise processes with zero mean and known covariance, described as follow:

$$
Q_{k}=E\left\{W_{k} W_{k}^{\mathrm{T}}\right\}=\left[\begin{array}{cc}
\sigma_{v_{k}}^{2} & 0 \\
0 & \sigma_{\phi_{k}}^{2}
\end{array}\right]
$$


Double Acoustic Communication Measurement. The position of follow AUV at time $k$ is $A_{k}\left(X_{k}^{A}, Y_{k}^{A}\right)$. The follow AUV calculates the position from time $k$ to $k+1$, at time $k+1$ the estimated position is $\hat{A}_{k+1}\left(\hat{X}_{k+1}^{A}, \hat{Y}_{k+1}^{A}\right)$. Then at time $k+1$, in Fig. 2 connect the two leader AUVs with the estimated position of follow AUV, there are two points $\hat{A}_{k+1}^{1}\left(\hat{X}_{k+1}^{1}, \hat{Y}_{k+1}^{1}\right)$ and $\hat{A}_{k+1}^{2}\left(\hat{X}_{k+1}^{2}, \hat{Y}_{k+1}^{2}\right)$. Where $P_{1}$ and $P_{2}$ are distances between leader and follow AUV in 2D plane, there are measured by acoustic devices. The $\hat{A}_{k+1}^{1}$ and $\hat{A}_{k+1}^{2}$ are double acoustic communication measurement.

Define $R_{k+1}^{1}$ is the distance between $T_{1}$ and $\hat{A}_{k+1}$ in 2D plane, $\hat{R}_{k+1}^{1}$ is the distance between $T_{1}$ and $\hat{A}_{k+1}^{1}$ in $2 \mathrm{D}$ plane.

$$
\begin{aligned}
& R_{k+1}^{1}=\left|T_{1}-\hat{A}_{k+1}\right| \\
& \hat{R}_{k+1}^{1}=\left|T_{1}-\hat{A}_{k+1}^{1}\right|
\end{aligned}
$$

From the Fig. 2 we can see the three points $\hat{A}_{k+1}, \hat{A}_{k+1}^{1}$ and $T_{1}$ are in one straight line, so we can get

Use Eq.(7) we can calculate $\hat{A}_{k+1}^{1}$.

$$
\left\{\begin{array}{c}
\frac{X_{k+1}^{1}-\hat{X}_{k+1}^{1}}{X_{k+1}^{1}-\hat{X}_{k+1}^{A}}=\frac{R_{k+1}^{1}}{\hat{R}_{k+1}^{1}} \\
\frac{Y_{k+1}^{1}-\hat{Y}_{k+1}^{1}}{Y_{k+1}^{1}-\hat{Y}_{k+1}^{A}}=\frac{R_{k+1}^{1}}{\hat{R}_{k+1}^{1}}
\end{array}\right.
$$

Define $R_{k+1}^{2}$ is the distance between $T_{2}$ and $\hat{A}_{k+1}$ in $2 \mathrm{D}$ plane, $\hat{R}_{k+1}^{2}$ is the distance between $T_{2}$ and $\hat{A}_{k+1}^{2}$ in $2 \mathrm{D}$ plane.

$$
\begin{aligned}
& R_{k+1}^{2}=\left|T_{2}-\hat{A}_{k+1}\right| \\
& \hat{R}_{k+1}^{2}=\left|T_{2}-\hat{A}_{k+1}^{2}\right|
\end{aligned}
$$

From the Fig. 2 we also can see the three points $\hat{A}_{k+1}, \hat{A}_{k+1}^{2}$ and $T_{2}$ are in one straight line, so we can get

$$
\left\{\begin{array}{c}
\frac{X_{k+1}^{2}-\hat{X}_{k+1}^{2}}{X_{k+1}^{2}-\hat{X}_{k+1}^{A}}=\frac{R_{k+1}^{2}}{\hat{R}_{k+1}^{2}} \\
\frac{Y_{k+1}^{2}-\hat{Y}_{k+1}^{2}}{Y_{k+1}^{2}-\hat{Y}_{k+1}^{A}}=\frac{R_{k+1}^{2}}{\hat{R}_{k+1}^{2}}
\end{array}\right.
$$

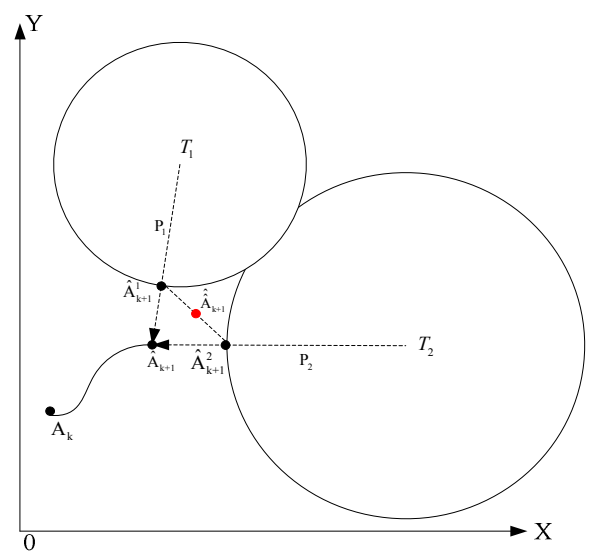

Figure 2. Double acoustic communication measurement

Then we define the middle point of $\hat{A}_{k+1}^{1}$ and $\hat{A}_{k+1}^{2}$ as the optimal estimated position of follow AUV.

$$
\left\{\begin{array}{l}
\hat{\hat{X}}_{k+1}^{A}=\frac{\hat{X}_{k+1}^{1}+\hat{X}_{k+1}^{2}}{2} \\
\hat{\hat{Y}}_{k+1}^{A}=\frac{\hat{Y}_{k+1}^{1}+\hat{Y}_{k+1}^{2}}{2}
\end{array}\right.
$$




\section{Simulations and Results}

To prove the efficiency of the algorithm, a simulation based on Matlab was studied. In Fig.3 two leader AUVs were programmed to execute a line flight, and they cruised at depth of $-20 \mathrm{~m}$. One follow AUV was commanded to execute a 45 degree line flight, another follow AUV was commanded to cruise a circle flight. They both were at depth of $-50 \mathrm{~m}$. Basic parameter includes the noise $\sigma_{V}^{2}=(0.5 \mathrm{kn})^{2}, . \sigma_{\phi}^{2}=(0.3 \mathrm{deg})^{2}$, and $\sigma_{P_{1}^{2}}^{2}=\sigma_{P_{2}^{2}}^{2}=(20 \mathrm{~m})^{2}$. The navigation results of the system are shown in

Fig.4 and Fig.6. The simulation was studied for one hundred times, and then we derived the error by Monte Carlo method. The error relative to the truth for both results is shown in Fig.5 and Fig.7. There is clear performance advantage from the result.

(i) Both the cooperative navigation methods, geometry triangulation method [8] and double acoustic measurement method, improve the navigation accuracy. Fig.5 and Fig. 7 show the errors of navigation.

(ii) Fig. 5 and Fig. 7 also show that the accuracy of both methods is close. The geometry triangulation method seems a little better than double measurement method, because in double acoustic measurement method the follow AUV must calculate its next time position by using its lower sensors before it calculates the relative position between leaders and itself. This adds the errors.

(iii) Fig.5 shows that the relative movement between the leader and follow AUV is a factor of navigation error. The formation of whole group must be considered.

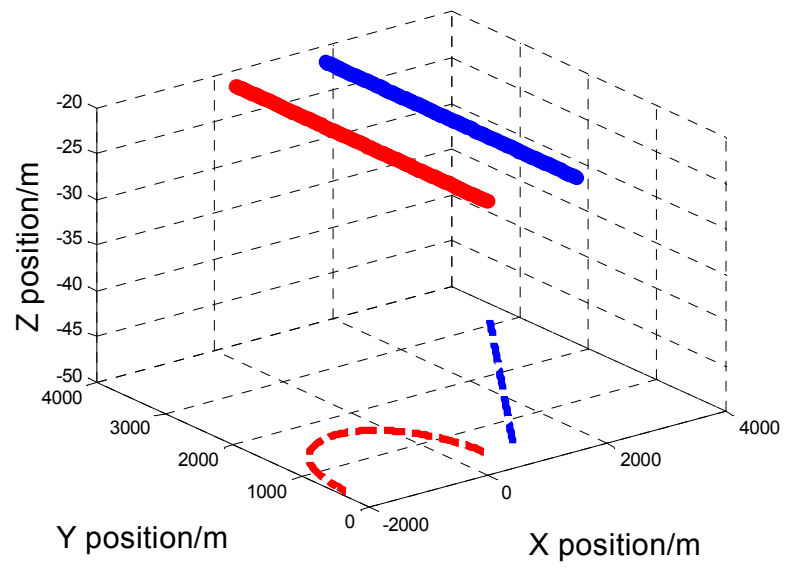

Figure 3. Position of leader and follow AUVs

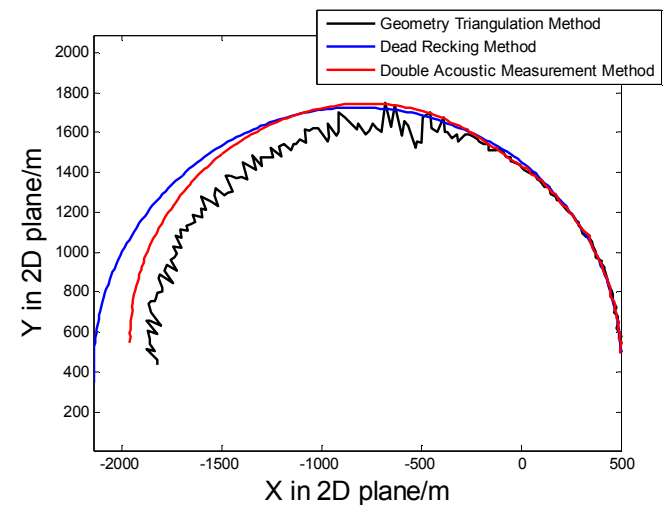

Figure 4. Results for circle flight using three methods

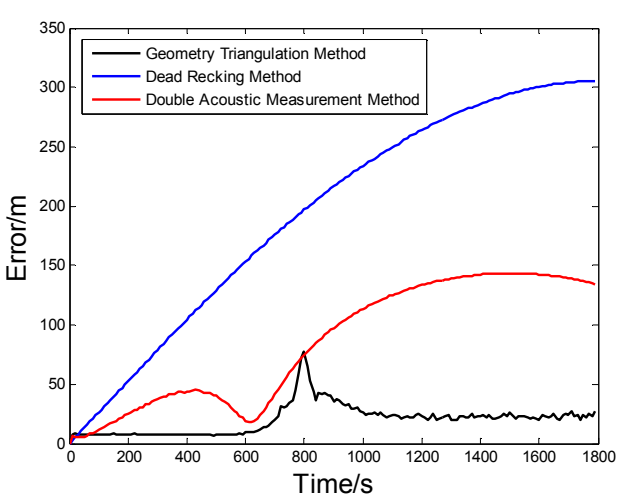

Figure 5. Errors for circle flight using three methods 


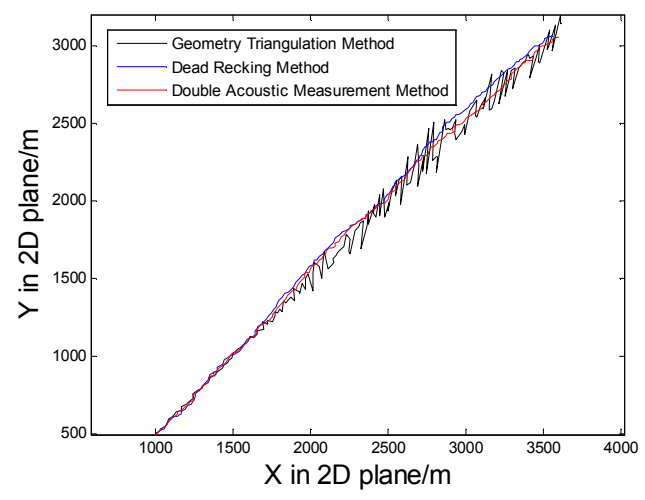

Figure 6. Results for line flight using three methods

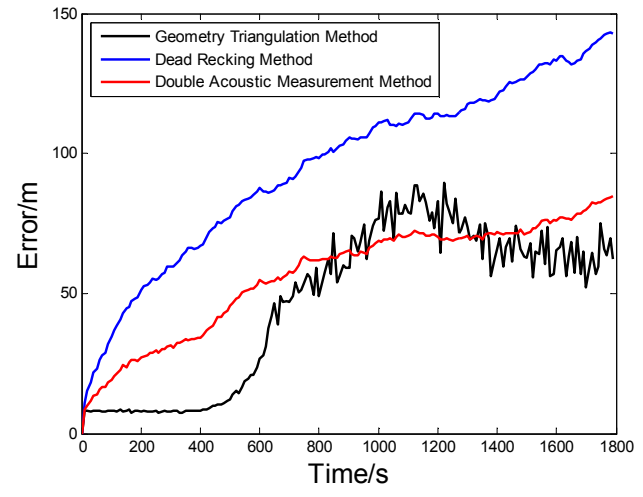

Figure 7. Errors for line flight using three methods

\section{Summary}

In this paper, a new approach to the AUV localization and navigation has been presented. The double acoustic measurement method was designed that optimally combines information from leader and follow AUV. Simulations show that the algorithm can perform more effectively than traditional method.

\section{Acknowledgment}

The authors would like to thank the associates of office for their constructive suggestions, as well as the supporting by the National Science Foundation of China (No. 51109179, 50979093) and the Foundation of Northwestern Polytechnical University(No. JC200905), Specialized Research Fund for the Doctoral Program of Higher Education (No. 20106102120057).

\section{References}

[1] L. Stutters, H. Liu, C. Tiltman, et al., Navigation Technologies for Autonomous Underwater Vehicles[J]. IEEE Transactions on Systems, Man, and Cybernetics-Part C: Applications and Reviews. 2008, 38(4), 581-589.

[2] W. S. Yan, D. M. Xu, J. Li and F. B. Zhang. Surver of Navigation Technology for Autonomous Underwater Vehicle[J]. F ire Control \& Command Control. 2004, 29(6): 11-15.

[3] A. I. Mourikis and S. I. Roumeliotis. Performance analysis of multirobot cooperative localization[J]. IEEE Transactions on Robotics, 2006, 22(4): 666-681

[4] A. I. Mourikis and S. I. Roumeliotis. Analysis of positioning uncertainty in reconfigurable networks of heterogeneous mobile robots[C]. IEEE International Conference on Robotics and Automation, New Orleans LA, 2004: 572-579

[5] D. K. Maczka, A. S. Gadre, D. J. Stilwell. Implementation of a Cooperative Navigation Algorithm on a Platoon of Autonomous Underwater Vehicles[C]. Proceedings of MTS/IEEE, 2007, pp. $730-735$

[6] J. Curcio, J. Leonard, J. Vaganay, A. Patrikalakis, A. Bahr, D. Battle, H. Schmidt, and M. Grund, Experiments in moving baseline navigation using autonomous surface craft[C]. Proceedings of MTS/IEEE, 2005, pp. 730-735.

[7] L. C. ZHANG, M. Y. LIU, D. M. XU, W. S. YAN. Cooperative Localization and Navigation for Multiple AUVs. Journal of System Simulation. 2008, 20(19): 5342-5344

[8] L. C. ZHANG, M. Y. LIU, D. M. XU, W. S. YAN. Cooperative navigation and localization for multiple UUVs. Journal of Marine Science And Application. 2009, 8(3): 216-221 